\title{
A GEOMETRIC APPROACH TO THE MULTIVARIATE MÜNTZ PROBLEM
}

\author{
ANDRÁS KROÓ
}

(Communicated by J. Marshall Ash)

\begin{abstract}
For a countable set $\Omega \subset \mathbb{R}^{n}$ denote by $P(\Omega)$ the space of polynomials spanned by $x^{\omega}, \omega \in \Omega \quad\left(x=\left(x_{1}, \ldots, x_{n}\right) \in \mathbb{R}^{n}, \omega=\left(\omega_{1}, \ldots, \omega_{n}\right) \in\right.$ $\left.\Omega, x^{\omega}=\prod_{i=1}^{n} x_{i}^{\omega_{i}}\right)$. In this paper we investigate the question of the density of $P(\Omega)$ in $C(K)$, the space of real valued continuous functions endowed with the supremum norm on compact set $K \subset \mathbb{R}^{n}$. In case $n=1$ the classical theorem of Müntz gives an elegant necessary and sufficient condition for density. This problem (closely related to the distribution of zeros of Fourier transforms) is much more complex in the multivariate setting. We shall present an extension of Müntz' condition to the case $n>1$ which will suffice for density. This, in particular, will enable us to construct "optimally sparse" lattice point sets $\Omega$ for which density holds.
\end{abstract}

\section{INTRODUCTION}

A famous result of Müntz asserts that given a sequence of real numbers $0<\lambda_{1}<\lambda_{2}<\cdots$ the functions $1, x^{\lambda_{1}}, x^{\lambda_{2}}, \ldots$ span $C[0,1]$ if and only if

$$
\sum_{k=1}^{\infty} \frac{1}{\lambda_{k}}=\infty
$$

This classical result inspired an intensive research of related questions. In particular, it was shown that (1) is also necessary for density in $C[a, b]$ whenever $0<a<b[1,7,11]$, Müntz-Jackson type results were obtained [3, 8, 9], the Müntz problem for rational functions was studied [2], and the multivariate Müntz problem was investigated $[4,5,10]$.

It may seem surprising that while some classical results of approximation theory (Weierstrass Theorem, Jackson Theorem, etc.) can be easily extended to multivariate cases, the multivariate Müntz problem appears to be very difficult. This fact can be explained by the known connection between Müntz-type problems and zero distribution of certain entire functions (the latter being of course much more complex for $n>1$ ). In the present paper we shall continue the investigation of the multivariate Müntz problem.

Received by the editors October 17, 1991 and, in revised form, September 1, 1992.

1991 Mathematics Subject Classification. Primary 41A10, 41 A63.

Supported by the Hungarian National Foundation for Scientific Research, Grant No. T4270. 
Denote by $\mathbb{N}^{n}, \mathbb{R}^{n}$, and $\mathbb{C}^{n}$ the set of integer, real, and complex $n$-tuples, respectively. (When $n=1$ the superscripts will be dropped.) In addition, $\mathbb{R}_{+}^{n}=$ $\left\{\left(a_{1}, \ldots, a_{n}\right) \in \mathbb{R}^{n}: a_{j} \geq 0,1 \leq j \leq n\right\}$. Furthermore, let $\Omega=\left\{\omega_{1}, \omega_{2}, \ldots\right\}$ be a countable subset of $\mathbb{R}^{n}$, and let $P(\Omega)=\operatorname{span}\left\{x^{\omega_{1}}, x^{\omega_{2}}, \ldots\right\}$ be the space of real polynomials with powers in $\Omega$ (as usual $x^{\omega}=\prod_{j=1}^{n} x_{j}^{a_{j}}$, where $x=$ $\left.\left(x_{1}, \ldots, x_{n}\right), \omega=\left(a_{1}, \ldots, a_{n}\right) \in \mathbb{R}^{n}\right)$. We shall say that $\Omega$ is separated, if $\inf \left\{\left\|\omega-\omega^{*}\right\|_{l_{1}}: \omega, \omega^{*} \in \Omega\right\}>0$. A model case of separated $\Omega$ is a lattice point set (i.e., $\Omega \subset \mathbb{N}^{n}$ ). Given a compact set $K \subset \mathbb{R}_{+}^{n}$ we denote by $C(K)$ the space of real continuous functions with supremum norm on $K$. Assuming that $P(\Omega) \subset C(K)$ we arrive at the following basic question: does the closure $\overline{P(\Omega)}$ of $P(\Omega)$ coincide with $C(K)$ ? The solution of the Müntz problem in case $n=1$ can be formulated in terms of the density function $\mu(\Omega, t)=$ $\#\left\{\omega \in \Omega:\|\omega\|_{l_{1}} \leq t\right\}$. It is easy to see that for $n=1$ equation (1) is equivalent to

$$
\int_{1}^{\infty} \frac{\mu(\Omega, t)}{t^{2}} d t=\infty
$$

When $n>1$ it is know $[5,6,10]$ that for separated $\Omega$ 's condition

$$
\limsup _{t \rightarrow \infty} t^{-n} \mu(\Omega, t)>0
$$

implies that $\overline{P(\Omega)}=C(K)$. Probably the weaker condition

$$
\int_{1}^{\infty} \frac{\mu(\Omega, t)}{t^{n+1}} d t=\infty
$$

suffices for density (for $n=2$ this was conjectured by Korevaar [5]). On the other hand if (3) fails for a given $\mu(t) \uparrow \infty$ such that $\mu_{0}(t):=\mu(t) / t^{n-1}$ is increasing, then setting $\Omega=\left\{\left(\lambda_{k}, r_{1}, \ldots, r_{n-1}\right): k, r_{j} \in \mathbb{N}\right.$ arbitrary $\}$ with $\lambda_{k}=\max \left\{r \in \mathbb{N}: \mu_{0}(r) \leq k\right\}$, we obtain a lattice point set $\Omega \subset \mathbb{N}^{n}$ such that $\mu(\Omega, t) \geq c \mu(t)$ but $\overline{P(\Omega)} \neq C(K)$ provided that Int $K \neq \varnothing$. (Since $\int_{1}^{\infty} \frac{\mu_{0}(t)}{t^{2}} d t<\infty$ the Müntz condition fails in the first variable.) Thus (3) should give the generally best possible sufficient condition for density.

On the other hand conditions $(*)$ and (3) are not necessary in general for density. Hellerstein [4] constructed very "sparse" sets $\Omega \subset \mathbb{R}_{+}^{n}$ asymptotic to the diagonal $\{(t, \ldots, t): t \in \mathbb{R}\} \subset \mathbb{R}^{n}$ for which density holds. (Evidently, this cannot be done with a lattice point set, so the question of constructing sparse lattice point sets for which density holds remained open.)

It is easy to see that (2) is necessary for density for every $n \geq 1$, whenever $K$ contains a nondegenerate segment $L$ whose continuation passes through the origin, and $\Omega \subset \mathbb{R}_{+}^{n}$. Indeed, assume that $\overline{P(\Omega)}=C(K)$ with $\Omega$ and $K$ as above. Let $u=\left(u_{1}, \ldots, u_{n}\right) \in \mathbb{R}^{n} \backslash\{0\}$ be the direction vector of $L$ and set $\widetilde{\Omega}=\left\{\left(r_{1}, \ldots, r_{n}\right) \in \Omega: r_{j}=0\right.$ if $\left.u_{j}=0,1 \leq j \leq n\right\}, G=\left\{\left\|w_{i}\right\|_{l_{1}}: \omega_{i} \in\right.$ $\widetilde{\Omega}\} \subset \mathbb{R}_{+}$. We may assume that $G=\left\{\lambda_{1}<\lambda_{2}<\cdots\right\}$. (If $\Omega$ has an accumulation point then (2) is trivial.) Evidently, the restriction of $P(\Omega)$ to $L$, which is spanned by $t^{\lambda_{i}}, i \in \mathbb{N}$, is dense in $C(L)$. Thus by the Müntz theorem $\sum_{i=2}^{\infty} 1 / \lambda_{i}=\infty$ yielding that $\int_{1}^{\infty} d \mu(\widetilde{\Omega}, t) / t=\infty$. Since $\mu(\widetilde{\Omega}, t) \leq \mu(\Omega, t)$, (2) should hold.

Hence condition (2) is necessary for density for every $n \geq 1$. It turns out (see, e.g., the Corollary below) that this necessary condition cannot be improved in general. 
Thus both the necessary condition (2) and the (conjectured) sufficient condition (3) are sharp in general and the gap between them cannot be narrowed (apart from the case $n=1$ when (2) and (3) coincide). Hence when (2) holds but $(*)$ fails for a given $\Omega$ the density of $P(\Omega)$ cannot be decided in term of $\mu(\Omega, t)$; here the geometry of $\Omega$ comes into play. In this paper we shall study $\Omega$ 's falling into the gap described above. We shall introduce a simple geometric property ("Müntz sets"), which appears to be a natural extension of the Müntz condition (1) for $n>1$ and which yields density. We shall see that Müntz sets fill in the gap between (2) and (3), that is for any $\mu(t)$ satisfying (2) there exists a Müntz set $\Omega \subset \mathbb{N}^{n}$ for which $\mu(\Omega, t) \leq \mu(t)$. Thus we construct "optimally sparse" lattice point sets for which density holds. (The method used by Hellerstein in [4] is not suitable for constructing lattice point sets.) Finally, we should mention that our Müntz condition is not necessary for density unless some additional restrictions are imposed on $\Omega$.

\section{NeW RESULTS}

Let us introduce the central notion for this paper of Müntz sets. We shall need some additional notation. For an arbitrary set $M \subset \mathbb{R}^{n}$ denote by $\operatorname{span} M$ the smallest plane in $\mathbb{R}^{n}$ containing $M$, i.e.,

$$
\operatorname{span} M=\left\{\sum_{i=1}^{k} a_{i} V_{i}: k \in \mathbb{N}, V_{i} \in M, a_{i} \in \mathbb{R}, \sum_{i=1}^{k} a_{i}=1\right\} .
$$

Furthermore, let $\operatorname{dim} M$ be the dimension of $\operatorname{span} M$, and denote by $d(M)$ the distance from $\operatorname{span} M$ to the origin: $d(M)=\inf \left\{\|m\|_{l_{2}}: m \in \operatorname{span} M\right\}$.

Definition 1. Let $\Omega \subset \mathbb{R}^{n}$. We shall now define Müntz sets relative to $\Omega$ in two steps.

$1^{\circ}$. Any point of $\Omega$ is a Müntz set (of dimension 0 ).

$2^{\circ}$. Let $\Omega \subset \Omega$ and $\operatorname{dim} \widetilde{\Omega}=k(1 \leq k)$. If $\widetilde{\Omega}$ contains $(k-1)$-dimensional Müntz sets $\Omega_{j} \subset \widetilde{\Omega}(j \in \mathbb{N})$ such that $\operatorname{span} \Omega_{j} \neq \operatorname{span} \Omega_{s}(s \neq j)$ and

$$
\sum_{j=1}^{\infty} \frac{1}{1+d\left(\Omega_{j}\right)}=\infty
$$

then $\widetilde{\Omega}$ is a Müntz set.

This defines Müntz sets of any dimension $k(0 \leq k \leq n)$. According to this definition points of $\Omega$ constitute the 0 -dimensional Müntz sets. A 1dimensional Müntz set (a "Müntz line") is a countable set of distinct points on a given line for which condition $(* *)$ holds. (In this case $(* *)$ is equivalent to the 1-dimensional Müntz condition (1).) Furthermore, a 2-dimensional Müntz set (a "Müntz plane") is a set of points on a given 2-dimensional plane containing distinct Müntz lines satisfying (**), and so on. It should be noted that the notion of Müntz sets is independent of the choice of basis in $\mathbb{R}^{n}$.

Example. Let $1=p_{1}<p_{2}<p_{3}<\cdots$ be the prime numbers. Set $\Omega=$ $\left\{\left(p_{k}, 2^{s}\left(p_{k}+p_{s}\right)\right): k, s \in \mathbb{N}\right\} \subseteq \mathbb{N}^{2}$. Then $\Omega$ is a 2-dimensional Müntz set. Indeed, it is easy to see that $L_{s}=\left\{\left(x, 2^{s} x+2^{s} p_{s}\right): x\right.$ a prime $\}$ is a Müntz line for any given $s \in \mathbb{N}$, where, in addition, $d\left(L_{s}\right) \leq p_{s}$. Hence $\Omega$ is a Müntz set. (In fact, we can replace $2^{s}$ by any integer $m_{s}$ in the definition of $\Omega$.)

One of our principal results is the next 
Theorem A. Let $K \subset \operatorname{Int} \mathbb{R}_{+}^{n}$ and assume that $\Omega \subset \mathbb{R}^{n}$ is an n-dimensional Müntz set $(n \geq 1)$. Then $\overline{P(\Omega)}=C(K)$.

This theorem can be considered as an extension of the sufficiency of Müntz' condition for $n>1$. Note that in the above theorem we assume that the compact $K$ is "strictly positive"; this restriction was needed for the Müntz property of $\Omega$ to be applied.

A standard application of the Hahn-Banach Theorem and Riesz Representation Theorem yields that if $\overline{P(\Omega)} \neq C(K)$ then there exists a measure $d \mu$ of total mass 1 such that, for every $\omega \in \Omega, \int_{K_{0}} e^{\langle x, \omega\rangle} d \mu(x)=0$, where $K_{0} \subset \mathbb{R}^{n}, K_{0}=\left\{\left(\ln t_{1}, \ldots, \ln t_{n}\right):\left(t_{1}, \ldots, t_{n}\right) \in K\right\}$. Thus setting

$$
f(z)=\int_{K_{0}} e^{-i\langle x, z\rangle} d \mu(x), \quad z=\left(z_{1}, \ldots, z_{n}\right) \in \mathbb{C}^{n},
$$

we obtain an entire function $f(z)$ of $n$ variables for which

$$
|f(z)| \leq k(f) e^{a(f)\left(\left|\operatorname{Im} z_{1}\right|+\cdots+\left|\operatorname{Im} z_{n}\right|\right)}
$$

and $f(i \omega)=0$ for $\omega \in \Omega(k(f), a(f)>0)$.

Let us denote by $\mathscr{B}^{n}$ the set of all entire functions of $n$-variables, which satisfy property (4) (constants $k(f), a(f)>0$ depend on the individual functions). In view of the above remarks Theorem A will follow easily from the next

Lemma. Let $f \in \mathscr{B}^{n}$ and assume that $f(i \omega)=0$ for every $\omega \in \Omega$, where $\Omega \subset \mathbb{R}^{n}$ is a Müntz set. Then $f(i \omega)=0$ for every $\omega \in \operatorname{span} \Omega$.

Note that the 1-dimensional version of this lemma $(n=1, \Omega$ is a 1 dimensional Müntz set) is equivalent to the well-known fact, that if $f \in \mathscr{B}^{1}$ and $f\left(i \lambda_{n}\right)=0$, where $\sum_{n=1}^{\infty} 1 / \lambda_{n}=\infty \quad\left(\lambda_{n} \in \mathbb{R}, \lambda_{n} \neq 0\right)$, then $f \equiv 0$ (see, e.g., $[7$, p. 26$])$.

In view of Theorem $A$ it is natural to ask what is the smallest possible rate of growth of the density function $\mu(\Omega, t)$ of an $n$-dimensional Müntz set $\Omega$. Of course, (2) should hold for such an $\Omega$, since this condition is necessary for density. According to our next theorem condition (2) provides the precise minimal rate of growth of density functions of Müntz sets.

Theorem B. Let $\mu(t)$ be a continuous positive increasing function on $\mathbb{R}_{+}$such that

$$
\int_{1}^{\infty} \frac{\mu(t)}{t^{2}} d t=\infty
$$

Then there exists an $n$-dimensional Müntz set $\Omega^{*} \subset \mathbb{N}^{n}$ such that $\mu\left(\Omega^{*}, t\right) \leq$ $\mu(t)$.

Note that in the above theorem we construct a Müntz set of lattice points, so, in particular, we obtain lattice point sets $\Omega$ satisfying $\overline{P(\Omega)}=C(K)$, which are optimally sparse (in terms of their density function).

Corollary. Let $\mu(t)$ be as in Theorem B. Then there exists $\Omega \subset \mathbb{N}^{n}$ such that $\mu(\Omega, t) \leq \mu(t)$ and $\overline{P(\Omega)}=C(K)$. 


\section{ProOFs}

Let us note that if $f \in \mathscr{B}^{n}, A_{0}$ is an $n \times n$ matrix and $c \in \mathbb{C}^{n}$ then $g(z)=f\left(A_{0} z+c\right) \in \mathscr{B}^{n}$.

Proof of the lemma. We shall verify our claim by induction on the dimension of Müntz sets $\Omega$. For 0 -dimensional Müntz sets the statement is trivial. So we assume that the Lemma holds for every $f \in \mathscr{B}^{n}$ and every Müntz set of dimension at most $k-1$, where $1 \leq k \leq n$. Let $\Omega \subset \mathbb{R}^{n}$ be a $k$-dimensional Müntz set and let $f \in \mathscr{B}^{n}$ be such that $f(i x)=0$ for $x \in \Omega$. We shall show first that without loss of generality it can be assumed that $\operatorname{span} \Omega=\mathbb{R}^{k} \subseteq \mathbb{R}^{n}$. Indeed, let $A_{0}$ be an $n \times n$ rotation matrix and $b \in \mathbb{R}^{n}$ such that $A_{0} \cdot \mathbb{R}^{k}+b=$ span $\Omega$. Since Müntz sets are basis invariant it is clear that $\Omega^{\prime}=A_{0}^{-1} \cdot(\Omega-b)$ is also a $k$-dimensional Müntz set and $\operatorname{span} \Omega^{\prime}=\mathbb{R}^{k}$. Furthermore, let $g(z)=$ $f\left(A_{0} z+i b\right)$, then $g \in \mathscr{B}^{n}$ and $g(i x)=0$ whenever $x \in \Omega^{\prime}$. Thus if we prove that $g(i x)=0$ for $x \in \operatorname{span} \Omega^{\prime}=\mathbb{R}^{k}$ then $f(i x)=0$ for $x \in \operatorname{span} \Omega$.

Thus we may assume that $\operatorname{span} \Omega=\mathbb{R}^{k}$. By definition of Müntz sets there exist $(k-1)$-dimensional Müntz sets $\Omega_{j} \subset \Omega(j \in \mathbb{N})$ such that $\operatorname{span} \Omega_{j} \neq$ span $\Omega_{s}(j \neq s)$ and $(* *)$ holds. Since $f(i x)=0$ for $x \in \Omega_{j}$ by the induction hypothesis

$$
f(i x)=0, \quad x \in \operatorname{span} \Omega_{j}(j \in \mathbb{N}) .
$$

Furthermore, since $\operatorname{span} \Omega_{j}$ is a $(k-1)$-dimensional plane in $\mathbb{R}^{k}$, we have for $j \in \mathbb{N}$

$$
\operatorname{span} \Omega_{j}=\left\{\left(t_{1}, \ldots, t_{k}\right) \in \mathbb{R}^{k}: a_{1, j} t_{1}+\cdots+a_{k, j} t_{k}+b_{j}=0\right\},
$$

where $a_{s, j}, b_{j} \in \mathbb{R}, \max _{1 \leq s \leq k}\left|a_{s, j}\right|=1$, and we may also assume that $a_{s, j}=$ -1 for some $1 \leq s \leq k$. Now we can organize all $\Omega_{j}$ 's into $k$ groups depending on which of coefficients $a_{s, j}$ equals $-1 \quad(1 \leq s \leq k)$. Then (**) should still hold for $\Omega_{j}$ 's belonging to at least one of these $k$ groups. Thus without loss of generality we may assume that $a_{k, j}=-1 \quad(j \in \mathbb{N})$, i.e.,

$$
\operatorname{span} \Omega_{j}=\left\{\left(t_{1}, \ldots, t_{k}\right) \in \mathbb{R}^{k}: t_{k}=a_{1, j} t_{1}+\cdots+a_{k-1, j} t_{k-1}+b_{j}\right\} .
$$

Let $B_{j, s}$ be the projection of $\operatorname{span} \Omega_{j} \cap \operatorname{span} \Omega_{s}$ to $\mathbb{R}^{k-1}$. Since span $\Omega_{j} \neq$ $\operatorname{span} \Omega_{s}$ if $j \neq s$ we have $\operatorname{dim} B_{j, s} \leq k-2$ for $j \neq s$. Set $B=\bigcup_{j \neq s} B_{j, s} \subset$ $\mathbb{R}^{k-1} . B$ is a countable union of $(k-2)$-dimensional subspaces of $\mathbb{R}^{k-1}$ and thus it is nowhere dense in $\mathbb{R}^{k-1}$. Hence it suffices to show that

$$
f\left(i t_{1}, \ldots, i t_{k-1}, i t\right)=0
$$

for every $\left(t_{1}, \ldots, t_{k-1}\right) \in \mathbb{R}^{k-1} \backslash B$ and $t \in \mathbb{R}$. Let $\left(t_{1}^{*}, \ldots, t_{k-1}^{*}\right) \in \mathbb{R}^{k-1} \backslash B$ and set $d_{j}=a_{1, j} t_{1}^{*}+\cdots+a_{k-1, j} t_{k-1}^{*}+b_{j}$. Then evidently all $d_{j}-s$ are distinct and we may also assume that they are different from zero (deleting $d_{j}=0$ ).

By (7) and (6)

$$
f\left(i t_{1}^{*}, \ldots, i t_{k-1}^{*}, i d_{j}\right)=0, \quad j \in \mathbb{N} .
$$

Since $\operatorname{span} \Omega_{j} \subset \mathbb{R}^{k}$ and $\operatorname{dim} \operatorname{span} \Omega_{j}=k-1$ it is easy to see that

$$
d\left(\Omega_{j}\right)=\operatorname{dist}\left(\operatorname{span} \Omega_{j}, 0\right)=\frac{\left|b_{j}\right|}{\sqrt{1+\sum_{s=1}^{k-1} a_{s, j}^{2}}} \geq \frac{\left|b_{j}\right|}{\sqrt{k}} .
$$


This and $(* *)$ imply that

$$
\sum_{j=1}^{\infty} \frac{1}{1+\left|b_{j}\right|}=\infty
$$

But

$$
\left|b_{j}-d_{j}\right|=\left|a_{1, j} t_{1}^{*}+\cdots+a_{k-1, j} t_{k-1}^{*}\right| \leq\left|t_{1}^{*}\right|+\cdots+\left|t_{k-1}^{*}\right|, \quad j \in \mathbb{N},
$$

hence by (9)

$$
\sum_{j=1}^{\infty} \frac{1}{\left|d_{j}\right|}=\infty
$$

Finally setting $g(z)=f\left(i t_{1}^{*}, \ldots, i t_{k-1}^{*}, z\right)\left(g \in \mathscr{B}^{1}\right)$ we have by $(8) g\left(i d_{j}\right)=$ $0, j \in \mathbb{N}$, where $d_{j}$ 's are distinct numbers satisfying condition (10). It is well known (see, e.g., [7, Lemma 2.2]) that condition (10) on zeros $i d_{j}$ of a function $g \in \mathscr{B}^{1}$ yields $g \equiv 0$. Since $\left(t_{1}^{*}, \ldots, t_{k-1}^{*}\right) \in \mathbb{R}^{k-1} \backslash B$ was chosen arbitrarily we arrive at the desired relation $f(i x)=0, x \in \mathbb{R}^{k}$. This completes the proof of the Lemma. Moreover, Theorem A now also follows.

In order to verify Theorem B we shall need two technical Propositions.

Let us denote by $\mathscr{M}$ the set of those increasing positive continuous functions on $\mathbb{R}_{+}$for which (5) holds.

Proposition 1. Let $\mu \in \mathscr{M}$. Then $\mu_{1}(t)=\min \{t, \mu(t)\} \in \mathscr{M}$. Moreover, there exists a $\mu_{2} \in \mathscr{M}$ such that $\mu_{2} / \mu$ is decreasing to 0 as $t \rightarrow \infty$.

Proof. Let us show that $\mu_{1} \in \mathscr{M}$. If $\mu(t)<t$ for all $t$ big enough then our claim is evident. Thus we may assume that $\mu\left(t_{k}\right) \geq t_{k}$ for $1<t_{1}<t_{2}<\cdots$ and $t_{k} \rightarrow \infty \quad(k \rightarrow \infty)$. Then we have

$$
\begin{aligned}
\int_{1}^{\infty} \frac{\mu_{1}(t)}{t^{2}} d t & \geq \sum_{k=2}^{\infty} \int_{t_{k-1}}^{t_{k}} \frac{\mu_{1}(t)}{t^{2}} d t \\
& \geq \sum_{k=2}^{\infty} \mu_{1}\left(t_{k-1}\right)\left(\frac{1}{t_{k-1}}-\frac{1}{t_{k}}\right) \geq \sum_{k=2}^{\infty} \frac{t_{k}-t_{k-1}}{t_{k}},
\end{aligned}
$$

where the last series diverges whenever $t_{k} \uparrow \infty$.

Now we construct a proper $\mu_{2}$. Set $\psi(x)=\int_{1}^{x} \frac{\mu(t)}{t^{2}} d t$. By (5) we have that $\psi(x) \uparrow \infty$. For the function $S(x)=\psi(\text { inv } \mu(x))^{1 / 2}$ (inv stands for the inverse function, $S(x) \uparrow \infty$ as $x \rightarrow \infty)$ there exists a positive continuous function $\varphi(x)$ such that

(i) $\varphi(x) \uparrow \infty, x \rightarrow \infty$;

(ii) $\varphi(x) \leq S(x), x \in \mathbb{R}_{+}$;

(iii) $\varphi(x) / x \downarrow 0$ as $x \rightarrow \infty$.

Now set $\mu_{2}(t)=\mu(t) / \varphi(\mu(t))$. Evidently, $\mu_{2}(t) \uparrow \infty$ and $\mu_{2}(t) / \mu(t) \downarrow 0$ as $t \rightarrow \infty$. Moreover, since $\varphi(\mu(t))$ increases we have

$$
\begin{aligned}
\int_{1}^{x} \frac{\mu_{2}(t)}{t^{2}} d t & \geq \frac{1}{\varphi(\mu(x))} \int_{1}^{x} \frac{\mu(t)}{t^{2}} d t=\frac{\psi(x)}{\varphi(\mu(x))} \\
& \geq \frac{\psi(x)}{\sqrt{\psi(x)}}=\sqrt{\psi(x)} \rightarrow \infty \quad(x \rightarrow \infty)
\end{aligned}
$$


Proposition 2. Let $\lambda_{k}$ be an increasing sequence of integers satisfying (1), $\tilde{\varphi}$ : $\mathbb{R}^{n-1} \rightarrow \mathbb{R}$ an arbitrary function, and $\mathbb{Q} \subseteq \mathbb{N}$ a countable set of integers. Then the set

$$
\Omega^{*}=\left\{\left(x_{1}, \ldots, x_{n}\right) \in \mathbb{N}^{n}: x_{j}=\lambda_{k} \prod_{i=1}^{j-1} r_{i}, r_{i} \in \mathbb{Q}, k \geq \tilde{\varphi}\left(r_{1}, \ldots, r_{n-1}\right)\right\}
$$

is an n-dimensional Müntz set.

Proof. Let $1 \leq s \leq n$ and choose an arbitrary $r_{1}^{*}, \ldots, r_{n-1}^{*} \in \mathbb{Q}$. Consider the set $\Omega\left(r_{1}^{*}, \ldots, r_{s-1}^{*}\right)=\left\{\left(x_{1}, \ldots, x_{n}\right) \in \mathbb{N}^{n}: x_{j}=\lambda_{k} \prod_{i=1}^{j-1} r_{i}\right.$, where $r_{i}=r_{i}^{*}$ if $1 \leq i \leq s-1, r_{i} \in \mathbb{Q}$ is arbitrary if $s \leq i \leq n-1$, and $\left.k \geq \tilde{\varphi}\left(r_{1}^{*}, \ldots, r_{s-1}^{*}, r_{s}, \ldots, r_{n-1}\right)\right\}, 1 \leq s \leq n$. This set results from $\Omega^{*}$ by fixing $r_{1}^{*}, \ldots, r_{s-1}^{*}$, and it reduces to $\Omega^{*}$ when $s=1$. Let us show that $\operatorname{dim} \Omega\left(r_{1}^{*}, \ldots, r_{s-1}^{*}\right)=n-s+1$. Choose distinct numbers $c_{1}, \ldots, c_{n-s+1} \in \mathbb{Q}$ and set

$$
v_{k}=\left(x_{1}^{(k)}, \ldots, x_{n}^{(k)}\right) \in \mathbb{R}^{n}, \quad x_{1}^{(k)}=1, x_{j}^{(k)}=\prod_{i=1}^{j-1} r_{i}^{(k)}, 2 \leq j \leq n,
$$

where $r_{i}^{(k)}=r_{i}^{*}$ if $1 \leq i \leq s-1$ and $r_{i}^{(k)}=c_{k}$ if $s \leq i \leq n-1 \quad(1 \leq$ $k \leq n-s+1)$. Obviously, $v_{1}, \ldots, v_{n-s+1}$ are linearly independent, and $\operatorname{span} \Omega\left(r_{1}^{*}, \ldots, r_{s-1}^{*}\right)$ contains lines with direction vectors $v_{1}, \ldots, v_{n-s+1}$ passing through the origin. Hence $\operatorname{dim} \Omega\left(r_{1}^{*}, \ldots, r_{s-1}^{*}\right)=n-s+1$. Moreover, $\operatorname{span} \Omega\left(r_{1}^{*}, \ldots, r_{s-1}^{*}\right) \subset H=\left\{\left(x_{1}, \ldots, x_{n}\right) \in \mathbb{R}^{n}: x_{j+1}=r_{j}^{*} x_{j}, 1 \leq j \leq\right.$ $s-1\}$. Since $H$ is an $(n-s+1)$-dimensional subspace of $\mathbb{R}^{n}$ it follows that $\operatorname{span} \Omega\left(r_{1}^{*}, \ldots, r_{s-1}^{*}\right)=H$. Now using induction on $s=n, n-1, \ldots, 1$ we can verify that $\Omega\left(r_{1}^{*}, \ldots, r_{s-1}^{*}\right)$ is an $(n-s+1)$-dimensional Müntz set. In particular, when $s=1$ this yields the statement of the Proposition. If $s=n$ then $\Omega\left(r_{1}^{*}, \ldots, r_{n-1}^{*}\right)=\left\{\lambda_{k} b: k \geq k_{0}\right\}$, where $b \in \mathbb{R}^{n}$ and $k_{0} \in \mathbb{N}$ are given. Thus by (1), $\Omega\left(r_{1}^{*}, \ldots, r_{n-1}^{*}\right)$ is a 1-dimensional Müntz set. Assume now that for an arbitrary choice of $r_{1}^{*}, \ldots, r_{s}^{*} \in \mathbb{Q}$ the set $\Omega\left(r_{1}^{*}, \ldots, r_{s}^{*}\right)$ is an $(n-s)$-dimensional Müntz set, and consider the set $\Omega\left(r_{1}^{*}, \ldots, r_{s-1}^{*}\right)$. Note that $\Omega\left(r_{1}^{*}, \ldots, r_{s-1}^{*}\right) \supset \Omega\left(r_{1}^{*}, \ldots, r_{s}^{*}\right)$ for any $r_{s}^{*} \in \mathbb{Q}, \operatorname{span} \Omega\left(r_{1}^{*}, \ldots, r_{s-1}^{*}, r_{s}^{*}\right) \neq$ $\operatorname{span} \Omega\left(r_{1}^{*}, \ldots, r_{s-1}^{*}, r_{s}^{* *}\right)$ whenever $r_{s}^{*} \neq r_{s}^{* *}$, and $d\left(\Omega\left(r_{1}^{*}, \ldots, r_{s}^{*}\right)\right)=0$ for every $r_{s}^{*} \in \mathbb{Q}$. Thus the $(n-s+1)$-dimensional set $\Omega\left(r_{1}^{*}, \ldots, r_{s-1}^{*}\right)$ contains a countable sequence of $(n-s)$-dimensional Müntz sets satisfying all requirements of Definition 1. Hence $\Omega\left(r_{1}^{*}, \ldots, r_{s-1}^{*}\right)$ is a Müntz set.

Proof of Theorem B. Given a $\mu(t) \in \mathscr{M}$ and using Proposition 1 we may assume that $\mu(t) \leq t$ and we can select a function $\varepsilon(t) \downarrow 0 \quad(t \rightarrow \infty)$ such that $\mu_{2}(t)=$ $\varepsilon(t) \mu(t) \in \mathscr{M}$. Set

$$
\begin{gathered}
\psi(t)=\left[\operatorname{inv} \mu_{2}(t)+t\right]+1, \quad \varphi(t)=\operatorname{inv}\left(\varepsilon^{-1 /(n-1)}(t)\right), \\
\Omega^{*}=\left\{\left(x_{1}, \ldots, x_{n}\right) \in \mathbb{N}^{n}: x_{j}=\lambda_{k} \prod_{i=1}^{j-1} r_{i}^{2}, \quad r_{i} \in \mathbb{N} \backslash\{1\}, k \geq \mu_{2}\left(\varphi\left(\prod_{i=1}^{n-1} r_{i}\right)\right)\right\},
\end{gathered}
$$

where $\lambda_{k}=\psi(k), k \in \mathbb{N}$. Let us show that $\left\{\lambda_{k}\right\}$ is an increasing sequence satisfying (1). Since $\mu_{2}$ is increasing (inv $\left.\mu_{2}(k+1)+k+1\right)-\left(\operatorname{inv} \mu_{2}(k)+k\right)>1$. 
Thus $\psi(k+1)>\psi(k)$. Furthermore, since $\mu(t) \leq t$, and hence $\mu_{2}(t)=O(t)$ we have by (5) $\int_{1}^{\infty} \frac{d \mu_{2}(t)}{t}=\infty$. Thus using inv $\mu_{2}(t) \geq c t$,

$$
\begin{aligned}
\sum_{k=1}^{\infty} \frac{1}{\lambda_{k}} & \geq \int_{1}^{\infty} \frac{d t}{\psi(t)} \geq \int_{1}^{\infty} \frac{d t}{\operatorname{inv} \mu_{2}(t)+t+1} \\
& \geq c_{1} \int_{1}^{\infty} \frac{d t}{\operatorname{inv} \mu_{2}(t)}=c_{1} \int_{t_{0}}^{\infty} \frac{d \mu_{2}(t)}{t}=\infty .
\end{aligned}
$$

Applying now Proposition 2 with $\mathbb{Q}=\left\{k^{2}: k \geq 1\right\}$ and $\tilde{\varphi}\left(r_{1}, \ldots, r_{n-1}\right)=$ $\mu_{2}\left(\varphi\left(\prod_{i=1}^{n-1} r_{i}\right)\right)$ we obtain that $\Omega^{*}$ is an $n$-dimensional Müntz set. It remains to estimate the growth of $\mu\left(\Omega^{*}, t\right)$. Points of $\Omega^{*}$ belong to the lines $L\left(r_{1}, \ldots, r_{n-1}\right)$ having direction vectors $\left(1, r_{1}^{2},\left(r_{1}, r_{2}\right)^{2}, \ldots,\left(r_{1} \cdots r_{n-1}\right)^{2}\right)$ $\left(r_{i} \geq 1\right)$ and passing through the origin. For given $r_{1}, \ldots, r_{n-1} \in \mathbb{N} \backslash\{1\}$ and $t \in \mathbb{R}_{+}$the number $k$ of points of $\Omega^{*} \cap L\left(r_{1}, \ldots, r_{n-1}\right)$ belonging to the $l_{1}$-ball in $\mathbb{R}^{n}$ of radius $t$ satisfies the relation $\lambda_{k}\left(r_{1} \cdot \cdots \cdot r_{n-1}\right)^{2} \leq t$, i.e., inv $\mu_{2}(k) \leq \psi(k) \leq t /\left(r_{1} \cdots \cdot r_{n-1}\right)^{2}$. Thus

$$
k \leq \mu_{2}\left(t /\left(r_{1} \cdots \cdot r_{n-1}\right)^{2}\right) .
$$

Moreover, if $r_{j}>\operatorname{inv} \varphi(t)$ for some $1 \leq j \leq n-1$ and $\left(x_{1}, \ldots, x_{n}\right) \in \Omega^{*} \cap$ $L\left(r_{1}, \ldots, r_{n-1}\right)$ then

$$
x_{1}=\lambda_{k} \geq \operatorname{inv} \mu_{2}(k) \geq \varphi\left(r_{1}, \cdots \cdot r_{n-1}\right)>t .
$$

Hence in order that $L\left(r_{1}, \ldots, r_{n-1}\right) \cap \Omega^{*}$ intersect the $l_{1}$-ball in $\mathbb{R}^{n}$ of radius $t$ it is necessary that $r_{j} \leq[\operatorname{inv} \varphi(t)], 1 \leq j \leq n-1$. This and (11) lead to the following estimate of the density function of $\Omega^{*}$ :

$$
\begin{aligned}
\mu\left(\Omega^{*}, t\right) & \leq \sum_{r_{1}=2}^{[\operatorname{inv} \varphi(t)]} \cdots \sum_{r_{n-1}=2}^{[\operatorname{inv} \varphi(t)]} \mu_{2}\left(\frac{t}{\left(r_{1} \cdots \cdot r_{n-1}\right)^{2}}\right) \\
& \leq \int_{1}^{\operatorname{inv} \varphi(t)} \cdots \int_{1}^{\operatorname{inv} \varphi(t)} \mu_{2}\left(\frac{t}{r_{1}^{2} \cdot \cdots \cdot r_{n-1}^{2}}\right) d r_{n-1} \cdots d r_{1} .
\end{aligned}
$$

Set $w=t / r_{1}^{2} \cdots \cdot r_{n-2}^{2}, w_{1}=w /(\operatorname{inv} \varphi(t))^{2}$, and $u=w / r_{n-1}^{2}$. Then

$$
\begin{aligned}
& \int_{1}^{\operatorname{inv} \varphi(t)} \mu_{2}\left(\frac{t}{r_{1}^{2} \cdot \cdots \cdot r_{n-1}^{2}}\right) d r_{n-1}=\int_{w}^{w_{1}} \mu_{2}(u)\left(-\frac{1}{2 w}\right)\left(\frac{w}{u}\right)^{3 / 2} d u \\
& =\frac{\sqrt{w}}{2} \int_{w_{1}}^{w} \mu_{2}(u) u^{-3 / 2} d u \leq \frac{\sqrt{w}}{2} \mu_{2}(w) \int_{w_{1}}^{w} u^{-3 / 2} d u \\
& =\sqrt{w} \mu_{2}(w)\left(\frac{1}{\sqrt{w_{1}}}-\frac{1}{\sqrt{w}}\right)=\mu_{2}(w)(\operatorname{inv} \varphi(t)-1) \leq \mu_{2}(w) \operatorname{inv} \varphi(t) .
\end{aligned}
$$

Thus we obtain from (12)

$$
\mu\left(\Omega^{*}, t\right) \leq \operatorname{inv} \varphi(t) \int_{1}^{\operatorname{inv} \varphi(t)} \cdots \int_{1}^{\operatorname{inv} \varphi(t)} \mu_{2}\left(\frac{t}{r_{1}^{2} \cdot \cdots \cdot r_{n-2}^{2}}\right) d r_{n-2} \cdots d r_{1} .
$$

We can repeat this process until we arrive at

$$
\mu\left(\Omega^{*}, t\right) \leq(\operatorname{inv} \varphi(t))^{n-1} \mu_{2}(t)=\mu_{2}(t) / \varepsilon(t)=\mu(t) .
$$


Thus by Theorem B one can construct Müntz sets with a minimal rate of growth of the density function. On the other hand the Müntz property is not necessary in general for density when $n>1$. (One such example is the set of all lattice points between curves $y=\sqrt{x}$ and $y=\sqrt{2 x}$; see [6].) Our next proposition shows that under additional restrictions property (**) of Müntz sets is necessary for density.

Proposition 3. Let $\Omega \subset \mathbb{R}^{n}$ be countable, $\operatorname{dim} \Omega=n(n \geq 1)$. Assume that $\Omega=\bigcup_{j \in T} \Omega_{j} \quad(T \subseteq \mathbb{N})$, where $\operatorname{dim} \Omega_{j}=n-1 \quad(j \in T)$ and

$$
\sum_{j \in T} \frac{1}{1+d\left(\Omega_{j}\right)}<\infty
$$

Then $\overline{P(\Omega)} \neq C(K)$ whenever $K \subset$ Int $K \neq \varnothing$.

When $n=1$ the above proposition reduces to the necessity of condition (1) for density in $C[a, b], 0<a<b$, proved by Korevaar and Luxemburg in [7]. The proof is essentially as in [7] with application of the multivariate Paley-Wiener Theorem. Therefore we give just an outline of the proof.

Proof. Let $\operatorname{span} \Omega_{j}=\left\{\left(t_{1}, \ldots, t_{n}\right) \in \mathbb{R}^{n}: a_{1, j} t_{1}+\cdots+a_{n, j} t_{n}=b_{j}\right\}$, where $\sum_{j=1}^{n} a_{k, j}^{2}=1$. Then $d\left(\Omega_{j}\right)=\left|b_{j}\right|$, hence by (13)

$$
\sum_{j \in T} \frac{1}{1+\left|b_{j}\right|}<\infty
$$

We may assume that $0=\left|b_{1}\right|=\cdots=\left|b_{s}\right|<\left|b_{s+1}\right| \leq\left|b_{s+2}\right| \leq \cdots$. Set for $z=\left(z_{1}, \ldots, z_{n}\right) \in \mathbb{C}^{n}$

$$
F(z)=\prod_{j=1}^{s}\left(\sum_{k=1}^{n} a_{k, j} z_{k}\right) \prod_{j \in T, j \geq s+1}\left(1+\frac{\left(\sum_{k=1}^{n} a_{k, j} z_{k}\right)^{2}}{b_{j}^{2}}\right) .
$$

Obviously, $F(i \omega)=0$ for every $\omega \in \Omega$. Moreover, similarly to [7, p. 29] one can show using (14) that

$$
|F(z)| \leq e^{\theta\left(\left|z_{1}\right|+\cdots+\left|z_{n}\right|\right)} \quad\left(z \in \mathbb{C}^{n}\right),
$$

where $\theta(t)$ is a positive increasing function such that $\int_{0}^{\infty} t^{-2} \theta(t) d t<\infty$. By [7, Theorem 3.1] there exists an entire function $f(u), u \in \mathbb{C}$ of exponential type $\tau \quad\left(\tau>0\right.$ arbitrary) such that $|f(x)| \leq e^{-\theta(n|x|)-\sqrt{|x|}}$ for $x \in \mathbb{R}$. Then choosing an arbitrary $a>0$ and setting $F_{1}(z)=F(z) \prod_{j=1}^{n} f\left(z_{j}\right), z \in \mathbb{C}^{n}$, we obtain an exponential function of type $\mathscr{D}_{a}\left(\mathscr{D}_{a}\right.$ being the $l_{1}$-ball in $\mathbb{R}^{n}$ of radius $1 / a)$ such that $F_{1} \in L_{2}\left(\mathbb{R}^{n}\right)$ and $F_{1}(i \omega)=0$ for every $\omega \in \Omega$. Then by the Paley-Wiener Theorem [13], $F_{1}$ is a Fourier transform of some function supported by $\mathscr{D}_{a}^{*}$, the $l_{\infty}$-ball in $\mathbb{R}^{n}$ of radius $a$. By making a shift we easily obtain a measure vanishing outside $K$ which annihilates $P(\Omega)$.

\section{ACKNOWLEDGMENT}

The author would like to express his gratitude to Professor Sz. Révész for many helpful conversations during the preparation of this manuscript. 


\section{REFERENCES}

1. J. A. Clarkson and P. Erdös, Approximation by polynomials, Duke Math. J . 10 (1943), 5-11.

2. G. Gierz and B. Shekhtman, $A$ duality principal for rational approximation, Pacific J. Math. 125 (1986), 79-92.

3. M. von Golitschek, Generalization of the Jackson approximation theorems in the sense of Ch. Müntz, Bull. Amer. Math. Soc. 75 (1969), 524-528.

4. S. Hellerstein, Some analytic varieties in the polydisc and the Müntz-Szasz problem in several variables, Trans. Amer. Math. Soc. 158 (1971), 285-292.

5. J. Korevaar, Müntz-type theorems for arcs and for $\mathbb{R}^{n}$, Second Edmonton Conf. on Approximation Theory (Z. Ditzian and A. Meir, eds.), 1982, pp. 199-225.

6. J. Korevaar and S. Hellerstein, Discrete sets of uniqueness for bounded holomorphic functions $f(z, w)$, Proc. Sympos. Pure Math., vol. 11, Amer. Math. Soc., Providence, RI, 1968, pp. 273-284.

7. W. A. J. Luxemburg and J. Korevaar, Entire functions and Müntz-Szasz type approximation, Trans. Amer. Math. Soc. 157 (1971), 23-37.

8. D. Leviatan, On the Jackson-Müntz theorem, J. Approx. Theory 10 (1974), 1-5.

9. D. J. Newman, A Müntz-Jackson theorem, Amer. J. Math 97 (1965), 940-944.

10. L. I. Ronkin, Some questions of completeness and uniqueness for functions of several variables, Functional Anal. Appl. 7 (1973), 37-45.

11. L. Schwartz, Étude des sommes d'exponentielles réelles, Actualités Sci. Indust., no. 959, Hermann, Paris, 1943.

12. G. Somorjai, A. Müntz-type problem for rational approximation, Acta Math. Acad. Sci. Hungar. 27 (1976), 197-199.

13. E. Stein and G. Weiss, Introduction to Fourier analysis on Euclidean spaces, Princeton Univ. Press, Princeton, NJ, 1971.

Mathematical Institute, Hungarian Academy of Sciences, Budapest, Reáltanoda u. 13-15, H-1053, HUNGARY 